\title{
Exploring phase coherence in a 2D lattice of Bose-Einstein condensates
}

\author{
Markus Greiner, Immanuel Bloch, Olaf Mandel, Theodor W. Hänsch*, and Tilman Esslinger \\ Sektion Physik, Ludwig-Maximilians-Universität, Schellingstr. 4/III, D-80799 Munich, Germany and \\ Max-Planck-Institut für Quantenoptik, D-85748 Garching, Germany
}

\begin{abstract}
Bose-Einstein condensates of rubidium atoms are stored in a two-dimensional periodic dipole force potential, formed by a pair of standing wave laser fields. The resulting potential consists of a lattice of tightly confining tubes, each filled with a $1 \mathrm{D}$ quantum gas. Tunnel-coupling between neighboring tubes is controlled by the intensity of the laser fields. By observing the interference pattern of atoms released from more than 3000 individual lattice tubes the phase coherence of the coupled quantum gases is studied. The lifetime of the condensate in the lattice and the dependence of the interference pattern on the lattice configuration are investigated.

03.75.Fi, 03.65.Nk, 05.30.Jp, 32.80.Pj
\end{abstract}

Bose-Einstein condensates trapped in periodic optical potentials provide a unique environment for experimental studies of a wide range of physical phenomena [1] [4]. Here we use an optical potential to create a two-dimensional periodic lattice filled with several thousand 1D quantum gases. In the $1 \mathrm{D}$ quantum gas the radial motion of the atoms is confined to zero point oscillations and transverse excitations are completely frozen out. In the degenerate limit, these systems are expected to show a remarkable physics not encountered in $2 \mathrm{D}$ and $3 \mathrm{D}$, for instance a continuous crossover from Bosonic to Fermionic behaviour as the density is lowered [5 9 . In our two-dimensional periodic array of quantum gases the coupling between neighboring lattice sites is controlled with a high degree of precision by changing the intensity of the optical lattice beams. After suddenly releasing the atoms from the trapping potential we observe the multiple matter wave interference pattern of several thousand expanding quantum gases. This allows us to study the phase coherence between neighboring lattice sites, which is remarkably long lived. Even for long storage times, when the phase coherence between neighboring lattice sites is lost and no interference pattern can be observed anymore, the radial motion of the atoms remains confined to zero point oscillations.

Similar to our previous work [10], almost pure BoseEinstein condensates with up to $5 \times 10^{5}{ }^{87} \mathrm{Rb}$ atoms are created in the $\left|F=2, m_{F}=2\right\rangle$ state. The cigar shaped condensates are confined in the harmonic trapping potential of a QUIC-trap [11] with an axial trapping frequency of $24 \mathrm{~Hz}$ and radial trapping frequencies of $220 \mathrm{~Hz}$. The lattice potential is formed by overlapping two perpendicular optical standing waves with the Bose-Einstein condensate as shown in Fig. 1. All lattice beams are derived from the output of a laser diode operating at a wavelength of $\lambda=852 \mathrm{~nm}$ and have spot sizes $w_{0}\left(1 / e^{2}\right.$ radius for the intensity) of approximately $75 \mu \mathrm{m}$ at the position of the condensate. The resulting potential for the atoms is directly proportional to the intensity of the interfering laser beams [12] and for the case of linearly polarized light fields it can be expressed by:

$$
\begin{aligned}
U(y, z)= & U_{0}\left\{\cos ^{2}(k y)+\cos ^{2}(k z)\right. \\
& \left.+2 \mathbf{e}_{1} \cdot \mathbf{e}_{2} \cos \phi \cos (k y) \cos (k z)\right\} .
\end{aligned}
$$

Here $U_{0}$ describes the potential maximum of a single standing wave, $k=2 \pi / \lambda$ is the magnitude of the wave vector of the lattice beams and $\mathbf{e}_{1,2}$ are the polarization vectors of the horizontal and vertical standing wave laser fields, respectively. The potential depth $U_{0}$ is conveniently measured in units of the recoil energy $E_{r}=\hbar^{2} k^{2} / 2 m$, with $m$ being the mass of a single atom. The time-phase difference between the two standing waves is given by the variable $\phi$ [13. In our setup this time-phase is measured interferometrically and controlled with a piezo-mounted mirror and a servo-loop. Furthermore, the intensity of the lattice beams is stabilized in order to ensure a constant potential depth during our measurements. The intensity pattern in the $y$ - $z$-plane extends along the $x$-direction, such that the resulting potential can be viewed as a lattice of narrow tubes with a spacing of $\lambda / 2$ between neighboring lattice sites. These tubes provide a tight harmonic confinement along the radial direction which leads to large trapping frequencies $\omega_{r} \approx \hbar k \sqrt{2 U_{0} / m}$. In our setup potential depths of up to $12 E_{r}$ are reached, resulting in a maximum radial trapping frequency of $\omega_{r} \approx 2 \pi \times 18.5 \mathrm{kHz}$. The confinement along the symmetry axis of a single tube is determined by the harmonic confinement of the magnetic trap and the confinement due to the gaussian intensity profile of the lattice laser beams. The trapping frequencies along the symmetry axis of a single lattice tube can be varied between $\omega_{a x} \approx 2 \pi \times 10 \mathrm{~Hz}-300 \mathrm{~Hz}$. The spontaneous scattering rate due to the lattice laser light is always less than $\Gamma_{s c}=0.02 \mathrm{~s}^{-1}$ and therefore negligible for our measurement times.

In order to transfer the atoms into the lattice potential, the laser power of the lattice beams is gradually increased in a linear ramp to its final strength within $40 \mathrm{~ms}$. The atoms are then held for a variable amount of time in the combined potential of the interfering laser beams and the magnetic trap. The number of occupied lattice sites can be estimated by counting the number of lattice 
sites within the Thomas-Fermi extension of the magnetically trapped condensate. For the above parameters we find that up to 3000 lattice sites are populated, with an average population of $\bar{N}_{i} \approx 170$ atoms per lattice site.

When the atoms are released from the combined potential of the optical lattice and the magnetic trap, the condensate wave functions on different lattice sites expand and interfere with each other. This interference pattern is imaged after a fixed expansion time using absorption imaging, with the imaging axis oriented along the $x$-axis and being parallel to the symmetry axis of the individual lattice tubes. The results are displayed in Fig. 2 for a 2D-lattice potential with a maximum potential depth of $12 E_{r}$ and orthogonal polarization vectors $\mathbf{e}_{1} \cdot \mathbf{e}_{2}=0$. In comparison, Fig. 3 shows the results for three different potential depths of the optical lattice and $1 \mathrm{D}$ vertical (z-axis), 1D horizontal ( $y$-axis) and 2D vertical+horizontal lattice configurations (orthogonal polarization vectors $\mathbf{e}_{1} \cdot \mathbf{e}_{2}=0$ ). Several important features can be seen on these images. First, discrete interference maxima are visible that are arranged in a regular structure. These interference maxima not only require a periodic density modulation of the atoms but also phase coherence of the condensate wave function throughout the lattice. They directly reveal the momentum distribution of the atoms in the lattice. Second, $s$-wave scattering spheres 11, 15 become more visible as the higher order momentum components are more strongly populated with increasing potential depth. These scattering spheres occur due to collisions between atoms in the separating momentum components after the trapping potential is switched off. The collision probability between atoms in the horizontal momentum components $\left|p_{y}\right|=2 \hbar k$ and the $|p|=0$ momentum component is high, due to the large extension of the condensate in the horizontal direction. This yields long interaction times and thus a high scattering probability. Along the vertical direction, the size of the condensate is almost an order of magnitude smaller and the interaction times are correspondingly shorter, resulting in a much lower scattering probability. Furthermore $s$-wave scattering spheres can also be seen in the diagonal direction due to collisions between the diagonal momentum components and the central $p=0 \hbar k$ momentum component. For a maximum trapping depth of $12 E_{r}$ all of these 8 scattering spheres (see Fig.2(b)) are clearly visible in Fig. 2(a).

The wave function of the Bose-Einstein condensate in the optical lattice can be expressed as a sum of localized wave functions on each lattice site. Such a localized wave function is described by a gaussian wave function for the ground state of the tightly confining radial axis of a single lattice tube, with radial widths as low as $90 \mathrm{~nm}$. Along the weakly confining axis of a lattice tube, the repulsive interactions between the atoms result in a parabolic Thomas-Fermi profile with a maximum radial width of $\approx 5 \mu \mathrm{m}$. The maximum chemical potential per lattice tube of $\mu \approx h \times 6 \mathrm{kHz}$ is then much smaller than the radial energy level spacing, confining the radial atomic motion to zero point oscillations.

In addition to a strong dependence of the visibility of the higher order momentum components on the localization of the wave function, we find a suppression of momentum components due to structural properties of the optical lattice. For a lattice configuration with orthogonal polarization vectors between the two standing waves for which the last term in the sum of eq.2 vanishes (see Fig. 1(a)), the first order diagonal momentum components with $|p|=\sqrt{2} \hbar k$ are completely suppressed, as can be seen in Fig. $4(\mathrm{~b})$. This is caused by a destructive interference between matter waves emitted from neighboring diagonal lattice planes and results in a vanishing geometrical structure factor of these momentum components 16]. If the lattice configuration is changed to parallel polarization vectors between the two standing waves, such that $\mathbf{e}_{1} \cdot \mathbf{e}_{2}=1$, and the timephase is set to $\phi=0$, the last term in eq. 2 modifies the geometry of the lattice (see Fig. 4(c)). For this lattice configuration the geometrical structure factor for the diagonal momentum components with $|p|=\sqrt{2} \hbar k$ does not vanish and they are clearly visible in the experiment (see Fig. $4(d)$ ).

Measuring the number of condensed atoms after a variable hold time in the optical lattice allows us to determine the lifetime of the condensate in the lattice. For this, the lattice is ramped up to its final strength within $40 \mathrm{~ms}$, then the atoms are held for a variable amount of time in the lattice after which the lattice potential is ramped down again in $40 \mathrm{~ms}$ and the remaining number of condensed atoms is measured after a ballistic expansion time using absorption imaging. The slow ramp speed ensures that the many-body wave function adjusts adiabatically to the changing optical potential. The results of this measurement are displayed in Fig. 5 for a lattice configuration with orthogonal polarization vectors $\mathbf{e}_{1} \cdot \mathbf{e}_{2}=0$ (see Fig. $4(\mathrm{a})$ ). The reduction of the lifetime of the condensate in the combined potential of the magnetic trap and the optical lattice compared to the lifetime in a pure magnetic trap is shown for three different lattice potential depths. We believe this reduced lifetime to be caused by remaining fluctuations of the lattice potential which lead to a dephasing of the neighboring condensates with time. This dephasing occurs faster with increasing potential depth, due to the corresponding strong reduction in the tunneling matrix element. In a band structure picture, the width of the energy bands decreases strongly with increasing potential depth of the lattice and external perturbations can then more easily lead to nonadiabatic transitions within a single band, resulting in a state where the atoms in a single lattice site remain within the ground state of this lattice site, but do not exhibit phase coherence to neighboring sites. Such dephased Bose-Einstein condensates are not recombined into a single condensate when the optical lattice poten- 
tial is turned off adiabatically. This strongly contributes to the measured decrease in condensate fraction with increasing storage time.

In order to verify this, we strongly reduce the tunneling matrix element by turning off the magnetic trapping field and exposing the atoms in a $12 E_{r}$ deep optical lattice to the linear gravitational potential. We then find that $2 \mathrm{~ms}$ after switching off the magnetic field we do not observe an interference pattern anymore after a sudden release of the atoms from the lattice potential. This indicates that phase coherence of the atoms throughout the lattice has been lost. In order to measure the band population of such a dephased Bose-Einstein condensate, we ramp down the optical potential in $2 \mathrm{~ms}$ after a $2 \mathrm{~ms}$ hold time in the pure optical potential. This ramp speed ensures that we are adiabatic with respect to the atomic motion in a single lattice site and preserve the band population. We then let the cloud of atoms expand for $12 \mathrm{~ms}$ and image the resulting momentum distribution. Atoms originating from the first energy band are then expected to obtain momenta that lie within the first Brillouin zone of the lattice [17]. The Brillouin zones of a two-dimensional Bravais lattice are shown in Fig. 6. (a), for which the first Brillouin zone is a square of width $2 \hbar k$. The experimental results in Fig. (b) display a pronounced squarelike momentum distribution of width $2 \hbar k$ which confirms that the atoms from a dephased condensate populate only the first energy band of the lattice. This directly proves that the atoms in a single lattice tube remain in the radial ground state of the system but no phase coherence between neighboring lattice sites is observed anymore. The same method is used to measure the band population after a variable storage time in the combined trapping field of the magnetic trap and the optical lattice. We find that for a $12 E_{r}$ deep lattice $60 \%$ of the initial number of atoms are still present after a storage time of $1 \mathrm{~s}$ and although no significant condensate fraction can

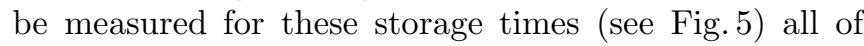
these atoms remain confined to the first energy band, i.e. they are still in the radial ground state of a lattice site. So far, we cannot distinguish whether the atoms in a single lattice tube are also in the axial ground state of this tube and only phase coherence between neighboring lattice sites has been lost or whether additional axial excitations in a single lattice tube are present. Our measurements therefore yield a lower limit for the phase coherence time of the atoms in the lattice and show that the radial motion in a lattice tube remains confined to zero point oscillations, i.e. is completely frozen out, even for long storage times.

In conclusions we have studied the phase coherence properties of a Bose-Einstein condensate stored in a twodimensional optical lattice potential. We have found that atoms from a Bose-Einstein condensate can be efficiently transferred into the ground state of the combined potential of the magnetic trap and the optical lattice consist- ing of several thousand tightly confining potential tubes, each filled with a 1D quantum gas. The phase coherence of atoms throughout the lattice was found to be remarkably long lived, while the radial motion of the atoms in a lattice tube remained frozen throughout our measurement time.

By decreasing the density of the atoms per lattice site we are now also able to enter the regime of quasicondensates and even that of a Tonks gas in which the fermionization of Bose systems [5.6] can be studied. Such a Tonks gas requires a very low number of atoms in a 1D geometry [7] and it seems promising to investigate this limit using the several thousand copies in our 2D optical lattice potential.

We would like to thank Wilhelm Zwerger and Martin Holthaus for stimulating discussions and Anton Scheich for experimental assistance during the construction of the experiment. We also acknowledge support by the Deutsche Forschungsgemeinschaft.

* also: Department of Physics, University of Florence, Italy

[1] B. P. Anderson and M. A. Kasevich, Science 281, 1686 (1998).

[2] C. Orzel et. al, Science 291, 2386 (2001).

[3] D. Jacksch et. al, Phys. Rev. Lett. 81, 3108 (1998).

[4] J. C. Bronski, L. D. Carr, B. Deconinck, and J. N. Kutz, Phys. Rev. Lett. 86, 1402 (2001).

[5] M. Girardeau, J. Math. Phys. (N. Y.) 1, 516 (1960).

[6] E. H. Lieb and W. Liniger, Phys. Rev. 130, 1605 (1962); E. H. Lieb, Phys. Rev. 130, 1616 (1963).

[7] M. Olshanii, Phys. Rev. Lett. 81, 938 (1998).

[8] D. S. Petrov, G. V. Shlyapnikov, and J. T. M. Walraven, Phys. Rev. Lett. 85, 3745 (2000).

[9] M. D. Girardeau, E. M. Wright, and J. M. Triscari, Phys. Rev. A, 63, 033601.

[10] M. Greiner, I. Bloch, T. W. Hänsch, and T. Esslinger, Phys. Rev. A 63, 031401 (2001).

[11] T. Esslinger, I. Bloch, and T. W. Hänsch, Phys. Rev. A 58, 2664 (1998).

[12] R. Grimm, M. Weidemüller, and Y. B. Ovchinnikov, Adv. At. Mol. Opt. Phys. 42, 95-170 (2000) and references therein.

[13] A. Hemmerich, D. Schropp, T. Esslinger, and T. W. Hänsch, Europhys. Lett. 18, 391 (1992).

[14] Y. B. Band, Marek Trippenbach, J. P. Burke, Jr., and P. S. Julienne, Phys. Rev. Lett, 84, 5462 (2000).

[15] A. P. Chikkatur et. al, Phys. Rev. Lett, 85, 483 (2000).

[16] See for example: N. W. Ashcroft and N. D. Mermin, Solid State Physics, Saunders College Publishing, 1976.

[17] A. Kastberg, W. D. Phillips, S. L. Rolston, and R. J. C. Spreeuw, Phys. Rev. Lett. 74, 1542 (1995). 


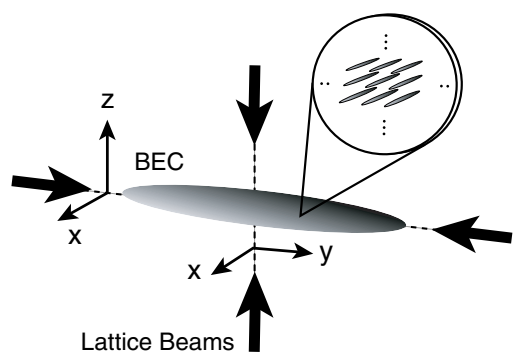

FIG. 1. Schematic setup of the experiment. A 2D lattice potential is formed by overlapping two optical standing waves along the horizontal axis ( $y$-axis) and the vertical axis ( $z$-axis) with a Bose-Einstein condensate in a magnetic trap. The condensate is then confined to an array of several thousand narrow potential tubes (see inset)

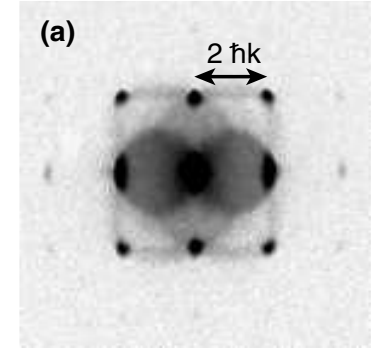

(b)

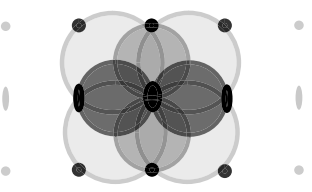

FIG. 2. (a) Average over 5 absorption images of released Bose-Einstein condensates that were stored in a $2 \mathrm{D}$ optical lattice potential. The maximum potential depth of the lattice was $12 E_{r}$ and the ballistic expansion time was set to $12 \mathrm{~ms}$. (b) Schematic image showing the expected discrete momentum states and the possible $s$-wave scattering spheres.

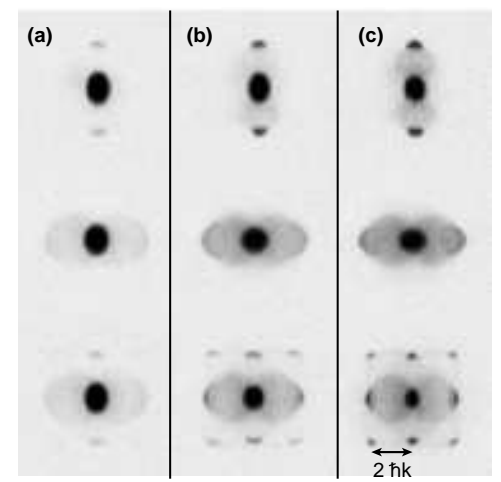

FIG. 3. Absorption images of Bose-Einstein condensates released from one-dimensional vertical (top row), one-dimensional horizontal (middle row) and two-dimensional horizontal+vertical (bottom row) lattice configurations. The images were taken for peak optical lattice depths of (a) $4 E_{r}$, (b) $8 E_{r}$, and (c) $12 E_{r}$. (a)

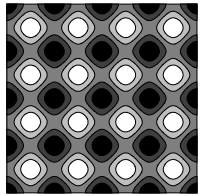

(b)

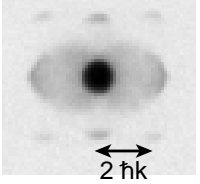

(c)

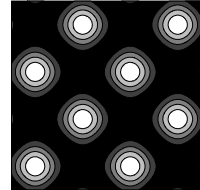

(d)

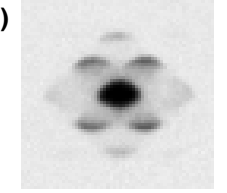

FIG. 4. Influence of the lattice configuration on the momentum distribution. For an optical lattice of (a) with orthogonal polarization vectors $\mathbf{e}_{1} \cdot \mathbf{e}_{2}=0$ the first diagonal momentum orders with $|p|=\sqrt{2} \hbar k$ are suppressed (b) due to their vanishing geometrical structure factor. In contrast, if $\mathbf{e}_{1} \cdot \mathbf{e}_{2}=0$ and $\phi=0$ as in (c), the resulting geometrical structure factor does not vanish for these momentum components and they are strongly visible (d).

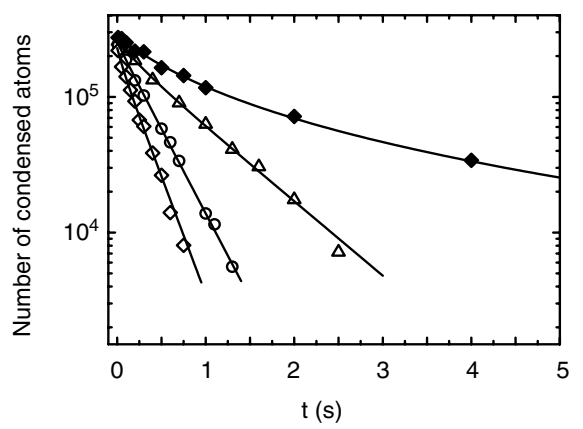

FIG. 5. Remaining number of condensed atoms after a variable hold time in the combined potential of the magnetic trap and the lattice potential (open datapoints) and in a pure magnetic trapping potential (solid diamonds). The maximum potential depth of the lattice was $4 E_{r}$ (open triangeles), $8 E_{r}$ (open circles) and $12 E_{r}$ (open diamonds), respectively. (a)

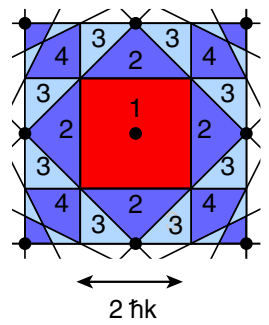

(b)

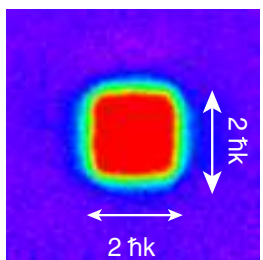

FIG. 6. (a) Reciprocal lattice and Brillouin zones for the two-dimensional Bravais lattice of Fig. 4(a). (b) False color image of the experimentally measured band population of a dephased Bose-Einstein condensate in a $12 E_{r}$ deep optical lattice where phase coherence between neighboring lattice sites has been lost. 